\title{
Letter to Neuroscience
}

\section{CHRONIC INTRASTRIATAL QUINOLINIC ACID PRODUCES REVERSIBLE CHANGES IN PERIKARYAL CALBINDIN AND PARVALBUMIN IMMUNOREACTIVITY}

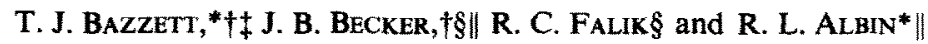 \\ *Department of Neurology, §Department of Psychology, †Reproductive Sciences Program, \\ NNeuroscience Program, The University of Michigan, 1103 E. Huron Street, Ann Arbor, \\ MI 48104-1687, U.S.A.
}

We recently reported the use of a chronic dialytic delivery system for intrastriatal administration of quinolinic acid in the rat. ${ }^{3,4}$ This system produces neurodegeneration with some characteristics similar to post mortem brain tissue from Huntington's disease patients, including reduced cytochrome oxidase staining, a decreased number of Nissl-stained neurons, and relative sparing of striatal NADPH-diaphorase containing neurons." The present findings show that chronic dialytic delivery of quinolinic acid also produces a Huntington's disease-like pattern of reduced calbindin and parvalbumin perikaryal immunoreactivity that is reversed in rats allowed four to eight weeks' recovery after cessation of quinolinic acid. Furthermore, cytochrome oxidase staining and the number of Nissl-stained cells were unchanged in the region of transient calbindin and parvalbumin immunoreactive perikaryal staining alterations. These results suggest that changes in calbindin and parvalbumin perikaryal immunoreactivity provide a relatively sensitive measure of quinolinic acid induced neurotoxicity. The reversible nature of reduced perikaryal immunoreactivity suggests a premorbid state of neurotoxicity, possibly marked by cellular redistribution of calbindin and parvalbumin.

Quinolinic acid acts as an agonist at $N$-methyl-Daspartate (NMDA) receptors, causing an increase in intracellular calcium concentration. ${ }^{20}$ To maintain intracellular ionic equilibrium, calcium can be sequestered by intracellular organelles or buffered by calcium binding proteins. ${ }^{1,2}$ Excessive calcium influx through the NMDA receptor complex results in excitotoxicity. ${ }^{10,15,21,22}$ Cells containing the calcium

†To whom correspondence should be addressed at the Department of Neurology/Neuroscience Laboratory Building.

Abbreviations: HD, Huntington's disease; NMDA, $N$ methyl-D-aspartate buffering protein, calbindin, are reduced in post mortem striatal tissue from all grades of Huntington's disease (HD) ${ }^{13,19}$ Cells containing parvalbumin, another calcium buffering protein, appear more resistant and are reduced only in tissue from advanced HD. ${ }^{9}$

In a previous report, Nissl staining showed that chronic intrastriatal dialytic delivery of $4 \mathrm{mM}$ quinolinic acid had no effect on neuronal density, while $15 \mathrm{mM}$ quinolinic acid produced a decrease in neuronal density that was restricted to an area approximately $400 \mu \mathrm{m}$ radial to the dialysis probe in the rat. ${ }^{4}$ In the present experiments, quantitation of Nisslstained neuron density revealed findings identical to the previous study for both 4 and $15 \mathrm{mM}$ concentrations of quinolinic acid. However, as shown in Fig. 1, perikaryal calbindin and parvalbumin immunoreactivity was significantly reduced throughout a region $400 \mu \mathrm{m}$ radial to the dialysis probe in animals receiving $4 \mathrm{mM}$ quinolinic acid (calbindin: control $170 \pm 9.55$ vs quinolinic acid $73.3 \pm 11.19$, $P<0.001$; parvalbumin: control $24.25 \pm 2.33$ vs quinolinic acid $14.3 \pm 2.68, P<0.005$; mean number of perikarya \pm S.E.M., two-tailed Student's paired $t$-test). Animals that received $15 \mathrm{mM}$ quinolinic acid showed reduced calbindin and parvalbumin perikaryal immunoreactivity throughout most of the striatum (Table 1, Fig. 2).

There was a dose-dependent effect of quinolinic acid on cytochrome oxidase staining. Analysis of brain selections containing the dialysis probe tract revealed that $15 \mathrm{mM}$ quinolinic acid produced a significantly greater area of decreased staining than did $4 \mathrm{mM}$ quinolinic acid (Veh $12.8 \pm 2.53 \mathrm{~mm}^{2}$ vs $4 \mathrm{mM} 27.1 \pm 1.22 \mathrm{~mm}^{2}$, mean \pm S.E.M., $P<0.001$; $4 \mathrm{mM} 27.1 \pm 1.22 \mathrm{~mm}^{2}$ vs $15 \mathrm{mM} 56.1 \pm 5.91 \mathrm{~mm}^{2}$, $P<0.001$; Student's unpaired $t$-test). For both doses, the decrease in perikaryal calbindin and parvalbumin immunoreactivity was apparent well beyond the area of decreased cytochrome oxidase staining. 

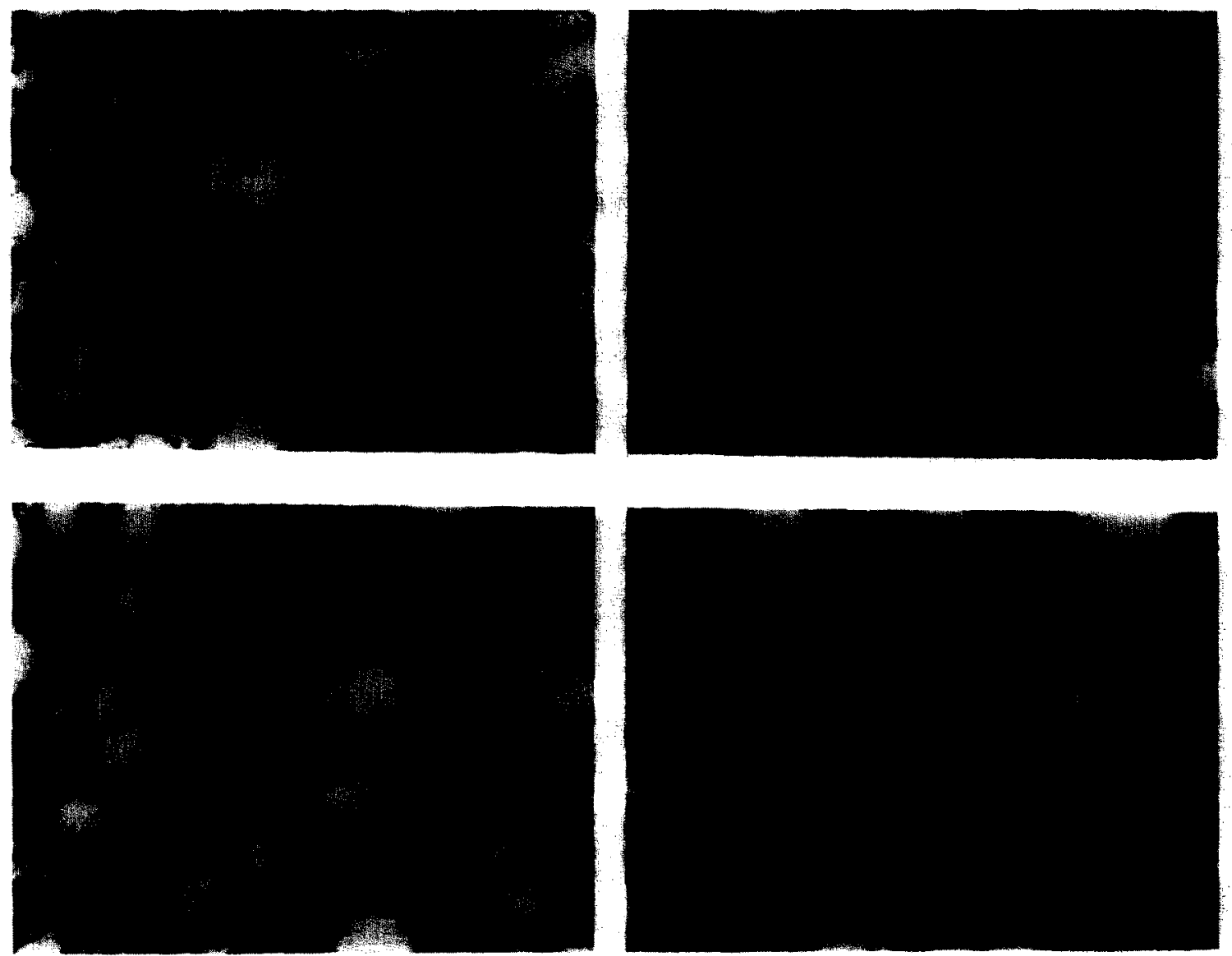

Fig. 1. Data from Experiment 1 showed that 21 days of $4 \mathrm{mM}$ quinolinic acid produced a significant decrease in calbindin and parvalbumin immunoreactive perikarya compared to control striata. Detailed methods for the dialytic delivery system are described elsewhere. ${ }^{3}$ Perikarya were analysed in a $1.02 \times 10^{-1} \mathrm{~mm}^{3}$ volume of striatal tissue $400 \mu \mathrm{m}$ radial from the necrotic core or a homologous region in the contralateral (control) striatum of 12 Sprague-Dawley rats (Harlan Sprague-Dawley, Inc. Indianapolis, IN). In the control striatum calbindin (A) and parvalbumin (C) immunoreactive staining is normal. Exposure to $4 \mathrm{mM}$ quinolinic acid for 21 days produced a significant decrease in calbindin (B) and parvalbumin (D) immunoreactive perikarya and an increase in neuropil staining. Calbindin and parvalbumin antibodies (Sigma) were diluted 1:2000 and immunohistochemical assays performed using Vectastain mouse IgG Elite ABC kit (Vector Labs, Inc.) according to manufacturer's instructions. There was no significant difference in the number of nissl-stained cells (not shown) in control (186.5 \pm 10.46$)$ vs quinolinic acid-exposed $(160.3 \pm 14.82)$ striata. Scale bar $=100 \mu \mathrm{m}$.

Table 1. Combined data from Experiment 2 showing a relatively rapid and sustained loss, and Experiment 3 showing the subsequent recovery, of calbindin and parvalbumin immunoreactive perikaryal staining in response to $15 \mathrm{mM}$ quinolinic acid

\begin{tabular}{ccccc}
\hline & \multicolumn{2}{c}{ Calbindin } & \multicolumn{2}{c}{ Parvalbumin } \\
& Quinolinic acid & Control & Quinolinic acid & Control \\
\hline Time course & & & & \\
5 days $(n=6)$ & $84.0 \pm 16.8^{* *}$ & $246.0 \pm 15.4$ & $9.2 \pm 2.4^{* *}$ & $25.8 \pm 2.7$ \\
10 days $(n=4)$ & $48.0 \pm 24.4^{*}$ & $212.1 \pm 26.1$ & $14.4 \pm 5.5$ & $25.0 \pm 2.5$ \\
15 days $(n=4)$ & $73.8 \pm 19.1^{*}$ & $251.0 \pm 44.4$ & $15.7 \pm 0.9^{*}$ & $29.3 \pm 2.4$ \\
21 days $(n=7)$ & $65.4 \pm 23.3^{* *}$ & $246.6 \pm 24.6$ & $11.5 \pm 2.0^{*}$ & $19.0 \pm 0.8$ \\
Recovered & & & & \\
4 weeks $(n=5)$ & $162.4 \pm 20.1$ & $209.6 \pm 30.5$ & $15.8 \pm 1.2$ & $16.2 \pm 1.8$ \\
8 weeks $(n=4)$ & $118.3 \pm 33.9$ & $193.3 \pm 36.3$ & $18.0 \pm 1.8$ & $19.8 \pm 3.3$ \\
\hline
\end{tabular}

Perikarya were analysed in a $1.02 \times 10^{-1} \mathrm{~mm}^{3}$ volume of tissuc from the ventral lateral striatum approximately $1 \mathrm{~mm}$ caudal from the necrotic core. Two-tailed paired Student's $t$-tests were used to determine significant changes in the number of calbindin and parvalbumin inmunoreactive perikarya between control and quinolinic acid exposed striata $\left(* P<0.05^{* *} P<0.005\right)$.

Cresyl Violet stained sections were also analysed in animals receiving 21 days $(n=7)$ administration. There was no significant difference in the number of Nissl-stained cells in control $(110.4 \pm 4.58)$ vs quinolinic acid exposed $(98.3 \pm 10.45)$ striata. 

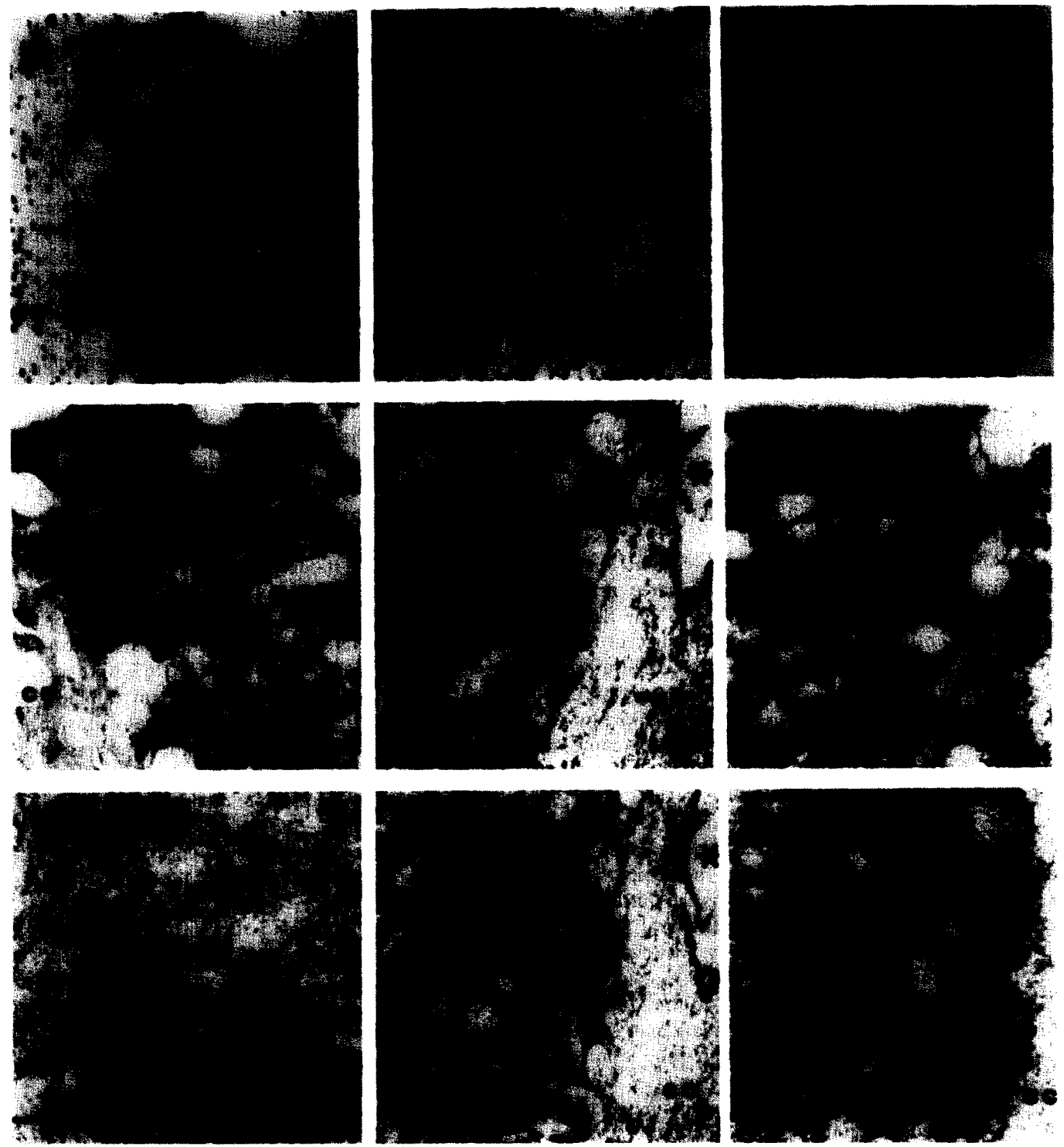

Fig. 2. Representative sections compare posterior lateral striatal tissue after: 21 days vehicle, 21 days $15 \mathrm{mM}$ quinolinic acid, and 21 days $15 \mathrm{mM}$ quinolinic acid plus four weeks recovery. Cresyl Violet staining was similar across the three groups. Calbindin and parvalbumin immunoreactive perikarya decreased after 21 days $21 \mathrm{mM}$ quinolinic acid, but not different after $15 \mathrm{mM}$ quinolinic acid plus four weeks' recovery, when compared to vehicle. Cresyl Violet staining after 21 days vehicle (A), 21 days quinolinic acid (B), 21 days quinolinic acid + four weeks of recovery (C). Calbindin immunoreactive staining 21 days vehicle (D), 21 days quinolinic acid (E), 21 days quinolinic acid + four weeks recovery (F). Parvalbumin immunoreactive staining 21 days vehicle $(G), 21$ days quinolinic acid $(H) 21$ days quinolinic acid + four weeks recovery (I). cc, Corpus callosum. *Blood vessel provides a landmark for comparison of consecutive $40-\mu \mathrm{m}$ sections from a brain exposed to 21 days quinolinic acid. Scale bar $=100 \mu \mathrm{m}$.

A time course evaluation of animals receiving $15 \mathrm{mM}$ quinolinic acid showed that the decrease in calbindin and parvalbumin perikaryal immunoreactivity occurs relatively rapidly (within five days), and is sustained throughout the 21 day course of quinolinic acid delivery (Table 1).

The ventrolateral region of the caudal striatum showed no change in the density of Nissl-stained cells 
but decreased perikaryal calbindin and parvalbumin immunoreactivity after 21 days of $15 \mathrm{mM}$ quinolinic acid compared to vehicle administration. If animals were allowed to survive either four or eight weeks after cessation of quinolinic acid administration, the number of immunoreactive calbindin and parvalbumin perikarya in this brain region was not different from the vehicle-treated striatum (Table 1, Fig. 2). The number of calbindin and parvalbumin immunoreactive perikarya were not different between fourand eight-week animals (Table 1).

The present results show that chronic dialytic delivery of quinolinic acid can decrease the number of calbindin and parvalbumin immunoreactive perikarya. The reversible nature of this effect suggests a change in production or distribution of these intracellular proteins. This hypothesis is further supported by the findings that there is no decrease in cytochrome oxidase staining or in the number of Nisslstained cells in regions exhibiting transient changes in calcium binding proteins.

Analysis of striata exposed to chronic dialytic delivery of quinolinic acid reveals three primary regions of specific neuronal reactions. The first region, a central necrotic core directly adjacent to the probe tract, is marked by inflammatory infiltrate and a lack of neurons. The second region, previously referred to as the transition zone, ${ }^{4,5}$ is marked by an absence of perikaryal staining for parvalbumin and calbindin, a reduction in the number of Nissl-stained cells and a decrease in cytochrome oxidase staining. Though many Nissl-stained neurons in the transition zone appear structurally intact, a decrease in cytochrome oxidase staining suggests metabolic impairment. Beyond the transition zone is the third region, marked only by a decrease in perikaryal calbindin and parvalbumin immunoreactivity. However, unlike the quinolinic acid-induced decrease in cytochrome oxidase and Nissl cell staining in the transition zone, decreased calbindin and parvalbumin staining in the region outside the transition zone is in part reversible.

There was also an increase in calbindin and parvalbumin neuropil staining throughout the striatum with both doses of quinolinic acid, and at all time points after $15 \mathrm{mM}$ quinolinic acid delivery. This increase was also apparent after acute quinolinic acid injections, ${ }^{23}$ and may have resulted from immunohistochemical neuropil staining caused by immunoglobulin extravasation. ${ }^{18}$

There are at least two explanations for the transient decrease in perikaryal calbindin and parvalbumin immunoreactivity following quinolinic acid administration. First, it is possible that some cells were excluded from analysis because they were not distinguished from increased neuropil staining. However, it is unlikely that a significant number of cells were excluded since the increase in neuropil staining at no time exceeded the intensity of staining scen in the vast majority of perikarya from the homologous region of the control striatum; and some immunoreactive neurons could be clearly seen against the increased background on the lesion side (Figs 1, 2).

Alternatively, the transient decrease in perikaryal calbindin and parvalbumin immunoreactivity may be caused by a change in distribution of these proteins within neurons. When stimulated, NMDA receptors allow an influx of calcium into dendritic processes where they are concentrated. ${ }^{6,22}$ Dendritic processes, which are capable of independent regulation of changes in intracellular calcium, ${ }^{8,14}$ could recruit calcium buffering proteins. Calbindin exhibits characteristics of a fast mobile calcium buffering protein, capable of diffusing from surrounding cytoplasm to local sites of calcium influx. ${ }^{17}$ Ferrante and colleagues ${ }^{7}$ found the intensity of calbindin immunoreactivity shifts to distal dendritic arbors in HD striatal tissue. A similar calbindin and parvalbumin shift in response to quinolinic acid could explain the observations of decreased perikaryal staining and increased neuropil staining.

It is also interesting to note that two weeks after acute intrastriatal quinolinic acid injection, levels of somatostatin and the glutamic acid decarboxylase isoforms GAD65 and GAD67 were decreased, ${ }^{16}$ whereas in a similar paradigm calbindin levels were unchanged. ${ }^{11}$ It is possible that exposure to quinolinic acid may produce long-term effects on production of some cellular components, while producing only temporary changes in production or distribution of others.

Calbindin-containing neurons appear less resistant than parvalbumin neurons to the neurodegenerative effects of HD..$^{9.19}$ The present results reveal that chronic quinolinic acid administration also reduces a greater percentage of calbindin- compared to parvalhumin-immunoreactive perikarya (Table 1). These results strengthen the contention that the pattern of neuronal vulnerability in HD is shared by striatal tissue exposed to chronic NMDA agonist administration.

The importance of assessing physiological neuronal states that precede cell death associated with neurodegenerative disorders has recently been discussed. ${ }^{12}$ In the present report, reversible quinolinic acid induced changes in calbindin and parvalbumin immunoreactivity may represent one such premorbid neuronal state. Further assessment of these changes may offer new insight into the neurodegenerative processes of HD.

Acknowledgements - This work was supported by NS00130, NS19613 and NS22157. Dr Bazzett was supported by training grants from The UM Reproductive Sciences Program (HD07048) and The UM Department of Neurology (NS07222-12). The authors would like to thank Kevin Kaatz, Brady Bustany and Elizabeth Vitarbo for their contributions to this project. We thank one of the anonymous reviewers for constructive criticism. 


\section{REFERENCES}

1. Abercrombie R. F. and Hart C. E. (1986) Calcium and proton buffering and diffusion in isolated cytoplasm from Myxicola axons. Am. J. Physiol. 250, C391-C405.

2. Baker P. F. and Umbach J. A. (1987) Calcium buffering in axons and axoplam of Loligo. J. Physiol. 383, 369-394.

3. Bazzett T. J., Becker J. B. and Albin R. L. (1991) A novel device for chronic intracranial drug delivery via microdialysis. J. Neurosci. Meth. 40, 1-8.

4. Bazzett T. J., Becker J. B., Kaatz K. W. and Albin R. L. (1993) Chronic intrastriatal dialytic administration of quinolinic acid produces selective neural degeneration. Expl Neurol. 120, 177-185.

5. Beal M. F., Kowall N. W., Swartz K. J., Ferrante R. J. and Martin J. B. (1989) Differential sparing of somatostatin-neuropeptide $\mathrm{Y}$ and cholinergic neurons following striatal excitotoxic lesions. Synapse 3, 38-47.

6. Benke T. A., Jones O. T., Collingridge G. L. and Angelides K. J. (1993) N-Methyl-D-aspartate receptors are clustered and immobilized on dendrites of living cortical neurons. Proc. natn. Acad. Sci. U.S.A. 90, 7819-7823.

7. Ferrante R. J., Kowall N. W. and Richardson E. P. (1991) Proliferative and degenerative changes in striatal spiny neurons in Huntington's disease: a combined study using the section-golgi method and calbindin D28k immunocytochemistry. J. Neurosci. 11, 3877-3887.

8. Guthrie P. B., Segal M. and Kater S. B. (1991) Independent regulation of calcium revealed by imaging dendritic spines. Nature 354, 76-80.

9. Harrington K. M. and Kowall N. W. (1991) Parvalbumin immunoreactive neurons resist degeneration in Huntington's disease striatum. J. Neuropathol. exp. Neurol. 50, 309.

10. Hartley D. M., Kurth M. C., Bjerkness L., Weiss J. H. and Choi D. W. (1993) Glutamate receptor-induced ${ }^{45} \mathrm{Ca}^{2+}$ accumulation in cortical cell culture correlates with subsequent neuronal degeneration. J. Neurosci. 13, $1993-2000$

11. Iacopino A., Chrisatkos S., German D., Sonsalla P. K. and Altar C. A. (1992) Calbindin-D $_{28-}$-containing neurons in animal models of neurodegeneration: possible protection from excitotoxicity. Molec. Brain Res. 13, 1992.

12. Isacson O. (1993) On ncuronal health. Trends Neurosci. 16, 306-308.

13. Kiyama H., Seto-Ohshima $A$, and Emson P. C. (1990) Calbindin $D_{28 k}$ as a marker for the degeneration of the striatonigral pathway in Huntington's disease. Brain Res. 525, 209-214.

14. Muller W. and Connor J. A. (1991) Dendritic spines as individual neuronal compartments for synaptic $\mathrm{Ca}^{2+}$ responses. Nature 354, 73-76.

15. Novelli A., Reilly J. A., Lysko P. G. and Henneberry R. C. (1988) Glutamate becomes neurotoxic via the $N$-methyl-D-aspartate receptor when intracellular energy levels are reduced. Brain Res. 451, 205-212.

16. Qin Y., Soghomonian J. J. and Chesselet M. F. (1992) Effects of quinolinic acid on messenger RNAs encoding somatostatin and glutamic acid decarboxylases in the striatum of adult rats. Expl Neurol. 115, 200-211.

17. Roberts W. M. (1993) Spatial calcium buffering in saccular hair cells. Nature 364, 74-76.

18. Schmidt-Kastner R., Meller D., Bellander B.-M., Stromberg I., Olson L. and Ingvar M. (1993). A one-step immunohistochemical method for detection of blood-brain barrier disturbances for immunoglobulins in lesioned rat brain with special reference to false-positive labelling in immunohistochemistry. J. Neurosci. Meth. 46, 121-132.

19. Seto-Ohshima A., Emson P. C., Lawson E., Mountjoy C. Q. and Carrasco L. H. (1988) Loss of matrix calcium-binding protein-containing neurons in Huntington's disease. Lancet 1, 1252-1255.

20. Tsuzuki K., Iino M. and Ozawa S. (1989) Change in calcium permeability caused by quinolinic acid in cultured rat hippocampal neurons. Neurosci. Lett. 105, 269-274.

21. Tymianski M., Wallace M. C., Spigelman I., Uno M., Carlen P. L., Tator C. H. and Charlton M. P. (1993) Cell-permeant $\mathrm{Ca}^{2+}$ chelators reduce early excitotoxic and ischemic neuronal injury in vitro and in vivo. Neuron 11, 221-235.

22. Tymianski M., Chariton M. P., Carlen P. L. and Tator C. H. (1993) Source specificity of early calcium neurotoxicity in cultured embryonic spinal neurons. J. Neurosci. 13, 2085-2104.

23. Waldvogel H. J., Faull R. L. M., Williams M. N. and Dragunow M. (1991) Differential sensitivity of calbindin and parvalbumin immunoreactive cells in the striatum to excitotoxins. Brain Res. 546, 329-335.

(Accepted 7 March 1994) 